\title{
Antioxidant Migration Studies in Chitosan Films Incorporated with Plant Extracts
}

\author{
Victor Gomes Lauriano Souza ${ }^{1}$, Patrícia Freitas Rodrigues ${ }^{2}$, Maria Paula Duarte ${ }^{1}$ and Ana Luísa Fernando ${ }^{1 *}$ \\ ${ }^{1}$ MEtRiCS, Departamento de Ciências e Tecnologia da Biomassa, Faculdade de Ciências e Tecnologia (FCT), Universidade NOVA de \\ Lisboa, Campus da Caparica, 2829-516 Caparica, Portugal \\ ${ }^{2}$ CENIMAT/I3N, Departamento de Ciências dos Materiais, Faculdade de Ciências e Tecnologia (FCT), Universidade NOVA de Lisboa, \\ Campus da Caparica, 2829-516 Caparica, Portugal
}

Received October 30, 2017; Accepted December 25, 2017

\begin{abstract}
The aim of this work was to develop an active biopolymer based on chitosan by incorporating natural antioxidants. Five essential oils (ginger, rosemary, sage, tea tree and thyme) and six hydro-alcoholic extracts (from ginger, green and black tea, kenaf leaves, rosemary and sage plants) were tested. Migration assays were carried out to evaluate the films' activity, and total phenolic content and antioxidant activity were monitored in the simulant during storage. Interaction between natural compounds and polymeric matrix was evaluated by FTIR spectroscopy. The diffusion of the phenolic compounds was not detected in the films incorporated with hydro-alcoholic extracts (HAEs), indicating their entrapment in the chitosan. Migration was observed in the films with essential oils (EOs), and biobased films incorporated with ginger, sage or rosemary essential oils, presented the highest diffusion and antioxidant activity in the simulant, highlighting their functionality and potential to be used as food active packaging material.
\end{abstract}

KEYWORDS: Active packaging, antioxidant activity, biopolymers, chitosan, phenolic content

\section{INTRODUCTION}

The most frequently used natural antioxidants are associated with secondary plant metabolites such as vitamins $\mathrm{C}$ and $\mathrm{E}$ (ascorbic acid and tocopherol, respectively) and numerous plant phytochemicals (such as phenolic acids, flavonoids, terpenes, carotenoids, volatile oils, among others) [1, 2]. Phenolic compounds are a large group of molecules (more than 8000 have been reported) with known antioxidant activity, which are widely dispersed in plants and are common in diets worldwide [1, 2]. The antioxidant activities of phenolic compounds have been extensively analyzed [1, 3]; they can participate in protection against the harmful effects of ROS (reactive oxygen species) and exhibit a wide range of biological effects. Therefore, these compounds have remarkable potential to be used as food preservatives either by direct incorporation as additives $[4,5]$ or into polymeric matrices as active packaging systems [5-10].

*Corresponding author: ala@fct.unl.pt

DOI: 10.7569/JRM.2018.634104
Oxidation is a naturally occurring process in biological systems, and in many cases is considered undesirable in food matrices, leading to their deterioration and resulting in the limitation of the product shelf life [11]. Active packaging with antioxidant properties is a novel technology approach to improve the preservation of food products, and the use of natural antioxidants as active compounds is driven by the trend of customer preference for more natural (less synthetic) products $[12,13]$.

Chitosan (poly- $\beta$-(1,4)-2-amino-2-deoxy-D-glucose) is a natural biopolymer obtained from the deacetylation of poly chitin ( $\mathrm{N}$-acetyl-D-glucosamine) found in exoskeletons of crustaceans and insects and on the cell walls of fungi and microorganisms [14]. This polysaccharide, the second most abundant in nature, is derived from renewable resources and due to its biodegradability, biocompatibility, ability to form membranes, gels, fibers, particles and resistant flexible films, and its nontoxicity, has potential to be used in food-grade films [15]. Moreover, biopolymers, including chitosan, and their applications, have shown an exponential growth in the past decades as a response to consumer demand for more environmentally friendly products [16]. 
Supplementation of the biopolymers by incorporation of antioxidant compounds is being considered as a strategy to improve the bioactivity of the materials [17]. In fact, there are some studies and reviews on the antioxidant activity of biobased films incorporated with natural antioxidants (e.g., $[6,8,18]$ ). Yet, the existing studies use pure compounds or natural antioxidants, individually, and only a few studies compare more than two antioxidants. The reviews on the subject are on antioxidant release systems, the various methods for incorporating antioxidant compounds into the package, the issues to be considered in packaging design, and the methods used to determine the oxidation protection effect of antioxidant active films. But, there are no relevant studies that compare the antioxidant activity of different active biobased films (namely those based on chitosan). In a previous work, Souza et al. [19] compared chitosan films incorporated with several natural antioxidants in terms of their physical properties. Among the tested extracts, black tea and green tea hydro-alcoholic extracts (HAEs) and sage, thyme and rosemary essential oils (EOs) were underlined as the most favorable due to the improved mechanical properties of the resulting films. However, in order to rank the different active films for the food packaging industry, it is also mandatory to evaluate the migration potential of the antioxidants to the food matrices or their barrier effect when incorporated into the biobased films. Thus, the aim of this work was to test the migration of antioxidant agents incorporated into the chitosan films, namely, five essential oils and six hydro-alcoholic extracts, into a fat food simulant.

\section{MATERIAL AND METHODS}

\subsection{Materials}

Chitosan with high molecular weight (31-37 kDa) and with a deacetylation degree of $75 \%$ was used as polymeric matrix and purchased from Sigma-Aldrich (Iceland). Active compounds were purchased in a local market: five different food-grade essential oils, used as purchased (Biover, Belgium), namely, Ginger EO (Zingiber officinale Roscoe); Rosemary EO (Rosmarinus officinalis L. ct. camphor); Sage EO (Salvia lavandulifolia Vahl); Tea Tree EO (Melaleuca alternifolia [Maiden \& Betche] Cheel); and Thyme EO (Thymus zygis Loefl. ex L. ct. linolol); and six different HAEs prepared from dried plants, namely, Black and Green Tea (Camellia sinensis [L.] Kuntze) from the Azores Islands (Gorreana, Portugal); Rosemary (Rosmarinus officinalis L.); Ginger (Zingiber officinale Roscoe); Sage (Salvia officinalis L.); and Kenaf leaves (Hibiscus cannabinus L.) variety Everglades 41, harvested in September 2005 before flowering (14th-17th October), from kenaf pilot fields in Caparica (latitude $38^{\circ} 40^{\prime} 03^{\prime \prime} \mathrm{N}$, longitude $9^{\circ} 12^{\prime} 8^{\prime \prime} \mathrm{W}$, altitude of $50 \mathrm{~m}$ ), Portugal.

\subsection{Chemicals}

All chemicals used were of analytical reagent grade. Acetic acid glacial, glycerol, gallic acid, and Tween 80 (polyethylene glycol sorbitan monolaurate) were purchased from Alfa Aesar (Germany), while FolinCiocalteu reagent, sodium carbonate anhydrous, sodium hydroxide, sodium nitrite, aluminum chloride and 2,2-azinobis(3-ethylbenzothiazoline-6-sulfonic acid) diammonium salt (ABTS) were obtained from Panreac (Spain). Ethanol absolute, 2,2-diphenyl-1picrylhydrazyl (DPPH), potassium peroxidisulfate, Trolox (6-hydroxy-2,5,7,8-tetramethylchroman-2carboxylic acid), and (+)-catechin hydrate ( $>96 \%$ ) were supplied by Sigma-Aldrich (Germany). All the water used was purified using a Milli-Q system (Millipore, Bedford, MA, USA).

\subsection{Hydro-Alcoholic Extracts (HAEs)}

Hydro-alcoholic extracts were obtained using solvent extraction assisted by ultrasonic force according to Turkmen et al. [20] and Pascoal et al. [4] with modifications. To enhance the superficial contact and maximize the phenolic content in the solvent media, prior to the extraction, all plants were ground into powder using an electric blender (ProfiCook model KSW 1021, Germany). To obtain each plant extract, $5 \mathrm{~g}$ of powder was weighed in an analytical balance (Mettler Toledo model AB204, Switzerland) and mixed in $50 \mathrm{~mL}$ of ethanol 50\% (v/v) using an Ultra-Turrax ${ }^{\circledR}$ disperser (IKA model T18, Germany). The mixture was kept refrigerated in the dark at $7{ }^{\circ} \mathrm{C} \pm 2{ }^{\circ} \mathrm{C}$ for $24 \mathrm{~h}$ and then submitted to ultrasonication for $30 \mathrm{~min} / 50 \mathrm{~Hz}$ in an ultrasonic bath (Selecta, Barcelona, Spain) at room temperature $\left(20^{\circ} \mathrm{C} \pm 2{ }^{\circ} \mathrm{C}\right)$. The system was centrifuged in conical tubes of $80 \mathrm{~mL}$ for $30 \mathrm{~min}$ at $4{ }^{\circ} \mathrm{C}$ with $10000 \times \mathrm{g}$ measured in the bottom of the tube using an angle rotor $(6 \times 80 / 85 \mathrm{~mL}$, NR12165, Sigma, Germany) and a refrigerated centrifuge (Sigma model $4 \mathrm{~K} 15$, Germany), the supernatant was removed, and the extraction was repeated once more. The combined supernatants from both extractions were filtered through Whatman $n^{\circ} 4$ filter paper, and the volume was corrected to $100 \mathrm{~mL}$ with ethanol $50 \%$ followed by concentration by rotary evaporator (Büchi Rotavapor model R-200, Switzerland) at $40{ }^{\circ} \mathrm{C}$ (Büchi heating bath model B-490, Switzerland) until a final volume of $25 \mathrm{~mL}$ (4 times reduction) was reached and, to avoid degradation, the extracts were stored at $-18{ }^{\circ} \mathrm{C}$ until analysis and use. 


\subsection{EO and HAE Characterization}

The HAEs and EOs used in the films were characterized by means of their total phenolic content (TPC) and antioxidant activity. Total phenolic content was determined according to the Folin-Ciocalteu method [21] with slight modifications. Briefly, $1 \mathrm{~mL}$ of diluted HAE or EO was mixed with $3 \mathrm{~mL}$ of Milli-Q water and $0.25 \mathrm{~mL}$ of Folin-Ciocalteu reagent. The mixture was incubated for $5 \mathrm{~min}$ at room temperature previous to the addition of $0.75 \mathrm{~mL}$ of sodium carbonate solution $5 \%(\mathrm{w} / \mathrm{v})$, and then it was agitated and stored in a dark chamber for $60 \mathrm{~min}$. The mixture absorbance was measured at $760 \mathrm{~nm}$ using a UV-Vis spectrophotometer (SPEKOL 1500, Analytik Jena, Germany). A calibration curve was constructed using gallic acid solutions from $0-120 \mathrm{mg} \cdot \mathrm{L}^{-1}$. Total phenolic content is expressed in $\mathrm{mg}$ gallic acid equivalent (GAE). $\mathrm{mL}^{-1}$ of EO or g dried plant (in the case of HAE).

Antioxidant activity was measured by two different methods: DPPH (2,2-diphenyl-1-picrylhydrazyl) free radical scavenging assay [22] and ABTS (2,2'-Azinobis(3-ethylbenzothiazoline-6-sulfonic acid) assays [23].

The ability to scavenge free radicals by the EOs and HAEs tested was measured by DPPH assay. An aliquot, or a diluted aliquot, of $1 \mathrm{~mL}$ of each natural antioxidant was mixed with $3 \mathrm{~mL}$ of $60 \mu \mathrm{mol} . \mathrm{L}^{-1} \mathrm{DPPH}$ ethanolic solution. The mixture was kept in the dark for $20 \mathrm{~min}$ and the absorbance was read at $517 \mathrm{~nm}$ using a UV-VIS spectrophotometer (SPEKOL 1500, Analytik Jena, Germany). DPPH, in its radical form, is purple and has a maximum absorbance at $517 \mathrm{~nm}$. Upon the activity of antioxidant, the compound is reduced (losing the purple color) and its absorption decreases. The results were calculated in two forms: by the percentage of inhibition using Equation 1 and as the Trolox equivalent antioxidant capacity (TEAC) using a calibration curve.

$$
\begin{aligned}
& \text { Scavenging }(\%) \\
& =\frac{(\text { Initial absorbance-Sampleabsorbance })}{\text { Initial absorbance }} \times 100
\end{aligned}
$$

ABTS assay was also carried out. Briefly, to generate the $\mathrm{ABTS}^{*+}$ cation equal parts of ABTS solution (7 $\left.\mathrm{Mm} . \mathrm{L}^{-1}\right)$ and potassium persulfate solution $\left(2.45 \mathrm{Mm} . \mathrm{L}^{-}\right.$ 1) were mixed and incubated for $16 \mathrm{~h}$ at room temperature in the dark. Then, ABTS $^{*+}$ cations generated were added to ethanol $80 \%$ until an absorbance of $0.7 \pm 0.05$ $\mathrm{nm}$ at $734 \mathrm{~nm}$ was reached. This was the $\mathrm{ABTS}^{*+}$ working solution used in the assay. An aliquot or a diluted aliquot of $0.5 \mathrm{~mL}$ of each natural antioxidant (EO or HAE) was added to $3.5 \mathrm{~mL}$ of the resulting ABTS*+ working solution, mixed and incubated for $6 \mathrm{~min}$ in the dark and the final absorbance was measured in a
UV-Vis spectrophotometer (SPEKOL 1500, Analytik Jena, Germany) at $734 \mathrm{~nm}$. A calibrating curve was constructed using standard solutions of Trolox. The results were expressed as Trolox equivalents ( $\mu \mathrm{mol}$ Trolox.mLL ${ }^{-1}$ EOs or g dry plant).

\subsection{Film Preparation}

Chitosan films were casted according to Souza et al. [19]. Film-forming dispersion (FFD) was prepared by dissolving $1.5 \%(\mathrm{w} / \mathrm{v})$ of chitosan in $1 \%(\mathrm{v} / \mathrm{v})$ of glacial acetic acid solution in ultrapure water with constant agitation using a magnetic stir plate during a 24 hour period at room temperature. Glycerol, the plasticizer, was incorporated into the proportion of 30\% (w/w chitosan) and the system was agitated for $5 \mathrm{~min}$ to complete homogenization. On treatments where EOs were added, Tween 80 at a level of $0.2 \%$ $\mathrm{w} / \mathrm{v}$ of essential oil was used as emulsifier [24]. The EO or HAE was incorporated into the system at the level of $1 \%(\mathrm{v} / \mathrm{v}$ FFS) and homogenized on a magnetic stir plate for an additional $5 \mathrm{~min}$ at room temperature. Degasification was performed with an ultrasonic bath for $5 \mathrm{~min}$. Films were casted in a glass mold $(18 \times 25 \mathrm{~cm})$ using $140 \mathrm{~mL}$ of the resulting solutions and dried for $72 \mathrm{~h}$ at room temperature. Chitosan films without incorporation of active compounds were used as control. After drying, films were peeled and stored protected from light until evaluation. Three repetitions were done for each EO and HAE tested, as well as with pristine chitosan films.

\subsection{Sample Characterization}

\subsubsection{Attenuated Total Reflectance Fourier Transform Infrared Spectroscopy (ATR-FTIR)}

The FTIR spectra of biobased films were from 4000 to $650 \mathrm{~cm}^{-1}$, resolution of $1 \mathrm{~cm}^{-1}$ in ATR mode using a Spectrum Two FTIR spectrometer (PerkinElmer, USA) according to Siripatrawan and Harte [25].

\subsubsection{Migration Assay}

Migration assay was carried out according to Lópezde-Dicastillo et al. [26]. The release of the active compounds from polymeric matrix was determined by the specific migration into $95 \%$ ethanol solution (fatty food simulant) at $37^{\circ} \mathrm{C} \pm 2{ }^{\circ} \mathrm{C}$ during 10 days. Square samples of $2.4 \mathrm{~cm}^{2}$ of each treatment were placed inside amber vials and $4 \mathrm{~mL}$ of the simulant was added, achieving an area-to-volume ratio of $6 \mathrm{dm}^{2} \cdot \mathrm{L}^{-1}$. The average thickness of the films produced $(61 \mu \mathrm{m})$ did not statistically change with the incorporation of 
the natural antioxidant extracts [14]. Therefore, the antioxidant properties of each film can be expressed based on sample surface area instead of sample weight. Periodically, total phenol content present in the simulant media and its antioxidant activity were determined by Folin-Ciocalteu method and DPPH/ ABTS assays, respectively, as described above in Section 2.4.

Complementary diffusion coefficients were also calculated from the plot of $\mathrm{M}_{\mathrm{Ft}} / \mathrm{M}_{\mathrm{P}, 0}$ versus $\mathrm{t}^{0,5}$ using initial migration data according to the model based on the Fick's second law described in Equation 2 [27]:

$$
\frac{M_{F, t}}{M_{P, 0}}=\frac{2}{L_{P}}\left(\frac{D t}{\pi}\right)^{0,5}
$$

where $\mathrm{M}_{\mathrm{F}: \mathrm{t}}$ (mg AGE) is the amount of migrant in the food (simulant) at time $t, \mathrm{M}_{\mathrm{P}, 0}$ (mg AGE) is the initial amount of migrant in the packaging film, $\mathrm{D}\left(\mathrm{cm}^{2} \cdot \mathrm{s}^{-1}\right)$ is the diffusion coefficient of migrant in the packaging film and $L_{p}(\mathrm{~cm})$ is the thickness of the packaging film.

\subsection{Statistical Analysis}

For each active compound used, three samples were analyzed and the results were expressed as mean values \pm standard deviation (SD). The results were analyzed using one-way analysis of variance (ANOVA) followed by Tukey's test with $a=0.05$. Statistical Analysis System (SAS), version 9.2, was used to treat the data.

\section{RESULTS AND DISCUSSION}

\subsection{EO and HAE Characterization}

Both EO and HAE incorporated into the chitosan filmforming solution demonstrated high content in total polyphenols with remarkable antioxidant activity (Table 1).

A good correlation was found between HAEs' antioxidant activity and HAEs' TPC, i.e., hydro-alcoholic extracts with highest TPC (green and black tea and rosemary HAEs) were also the ones that presented the highest antioxidant activity, and the ones with the lowest TPC (kenaf and ginger HAEs) also presented the lowest antioxidant activity. A comparison of the findings with published data is difficult due to the different types of extraction methods used [28]. Kenaf, ginger and green and black tea HAEs showed smaller TPC than what was reported [4, 20, 29-32]. In opposition, rosemary and sage HAEs were higher than those reported in the literature for similar extraction processes [2]. But differences can be attributed to the different time of contact, the different energy/ temperature employed or by the use of a solvent to which polyphenols have higher affinity (e.g., methanol) $[20,29,31]$.

The antioxidant activity of the EOs was correlated with their TPC for ginger and thyme, but not for the remaining EOs studied. Tea tree was the richest in phenolic content but the poorest in terms of antioxidant activity. Rosemary EOs showed a low TPC in

Table 1 Total phenolic content and antioxidant activity of essential oils and hydro-alcoholic extracts incorporated into chitosan films.

\begin{tabular}{|l|c|c|c|}
\hline Sample & $\begin{array}{c}\text { Total phenolic content } \\
(\mathbf{m g} \text { gallic acid/g dry plant) }\end{array}$ & $\begin{array}{c}\text { DPPH assay } \\
(\mu \text { molTrolox/g dry plant) }\end{array}$ & $\begin{array}{c}\text { ABTS assay } \\
(\mu \mathrm{molTrolox} / \mathbf{g} \text { dry plant) }\end{array}$ \\
\hline Green tea hydro alcoholic extract & $68 \pm 1^{\mathrm{a}}$ & $904 \pm 36^{\mathrm{a}}$ & $1636 \pm 77^{\mathrm{a}}$ \\
\hline Black tea hydro alcoholic extract & $30 \pm 1^{\mathrm{b}}$ & $592 \pm 32^{\mathrm{b}}$ & $920 \pm 20^{\mathrm{b}}$ \\
\hline Kenaf hydro alcoholic extract & $11.8 \pm 0.2^{\mathrm{d}}$ & $160 \pm 3^{\mathrm{e}}$ & $147 \pm 2^{\mathrm{e}}$ \\
\hline Ginger hydro alcoholic extract & $7.4 \pm 0.02^{\mathrm{d}}$ & $43.6 \pm 0.8^{\mathrm{f}}$ & $199 \pm 0^{\mathrm{e}}$ \\
\hline Rosemary hydro alcoholic extract & $33 \pm 0^{\mathrm{b}}$ & $476 \pm 14^{\mathrm{c}}$ & $508 \pm 30^{\mathrm{c}}$ \\
\hline Sage hydro alcoholic extract & $20.8 \pm 0.4^{\mathrm{c}}$ & $306 \pm 8^{\mathrm{d}}$ & $347 \pm 8^{\mathrm{d}}$ \\
\hline Sample & $\begin{array}{c}\text { Total phenolic content } \\
(\mathrm{mg} \text { gallic acid/mL) }\end{array}$ & $\begin{array}{c}\text { DPPH assay } \\
(\mu \mathrm{molTrolox} / \mathrm{mL})\end{array}$ & $\begin{array}{c}\text { ABTS assay } \\
(\mu \mathrm{molTrolox} / \mathrm{mL})\end{array}$ \\
\hline Ginger essential oil & $16 \pm 1^{\mathrm{b}}$ & $2.83 \pm 0.06^{\mathrm{a}}$ & $10.0 \pm 0.3^{\mathrm{a}}$ \\
\hline Rosemary essential oil & $8.9 \pm 0.8^{\mathrm{c}}$ & $2.98 \pm 0.09^{\mathrm{a}}$ & $14.8 \pm 0.1^{\mathrm{a}}$ \\
\hline Sage essential oil & $7.0 \pm 0.8^{\mathrm{cd}}$ & $0.98 \pm 0.02^{\mathrm{b}}$ & $11.9 \pm 0.2^{\mathrm{a}}$ \\
\hline Tea tree essential oil & $23 \pm 1^{\mathrm{a}}$ & $0.30 \pm 0.08^{\mathrm{d}}$ & $3.9 \pm 0.2^{\mathrm{b}}$ \\
\hline Thyme essential oil & $6.4 \pm 0.3^{\mathrm{d}}$ & $0.72 \pm 0.02^{\mathrm{c}}$ & $4.4 \pm 0.1^{\mathrm{b}}$ \\
\hline
\end{tabular}

a-f: Different superscripts within the same column, within essential oils or hydro alcoholic extracts, indicate significant differences among samples $(\mathrm{p}<0.05)$ 
comparison to tea tree and ginger, but its antioxidant activity was the highest. Sage EO presented a similar TPC to rosemary EO, which was correlated with the antioxidant activity measured through the DPPH assay. Yet, sage EO results by the ABTS assay showed a significant high antioxidant activity. This can be explained by its different chemical composition, since the antioxidant activity depends not only on the concentration of phenolic compounds but also its phenolic profile [29]. Results in essential oil TPC and antioxidant activity presented in this study show differences to those presented in the literature. This can be due to several factors, such as geographical location, season, environmental conditions, nutritional status of the plants, the solvent used to dilute the EOs, among other factors [33].

\subsection{Migration Assay}

Only chitosan films incorporated with EO showed migration into fatty simulant media after 10 days of storage at $37^{\circ} \mathrm{C} \pm 2{ }^{\circ} \mathrm{C}$. HAE extracts did not diffuse toward simulant, thus no phenolic content or antioxidant activity was detected by Folin-Ciocalteu method and DPPH/ABTS assays, respectively. Apparently, the compounds present in HAE extracts had a stronger affinity with the polymeric matrix than with the simulant used (fatty food simulant), resulting in lower solubility and undetectable diffusion by the methods used. Good entrapment of active compounds within polymer matrix reduces its release to simulant [34]. According to a previous study [19], films incorporated with HAEs exhibited higher solubility in water than chitosan incorporated with EOs, and a lower swelling degree, suggesting lower free volume between knots to swell with ethanol, thus lower relaxation of the matrix and release of active compounds. The incorporation of only $1 \%(\mathrm{v} / \mathrm{v})$ of HAE in the chitosan and its hydrophilic character might also explain the results obtained. Perhaps novel formulations with higher content of hydro-alcoholic extracts may result in an observed diffusion to the simulant, as reported previously by López-de-Dicastillo et al. [26], who incorporated more than $5 \%$ of green tea extracts in ethylenevinyl alcohol copolymer (EVOH) films.

The good interaction between HAE and chitosan polymer is an advantage since the polyphenols of the extracts incorporated can also protect the thin film produced against oxidation [17]. Furthermore, as the final purpose of the material is to be used as active food packaging, when in direct contact with foods (complex matrices with either hydrophobic and hydrophilic parts), a different behavior is expected, with potential diffusion of the active compounds toward the products packaged resulting in gradual preservation throughout their shelf life. Also, the polyphenols entrapped in the chitosan chains can improve the barrier properties of the material, thus retarding possible contact of the food packaged with water, oxygen or light since the bioactive compounds represent a shield and a reaction media prior to contact with food.

Chitosan films incorporated with EOs exhibited an "exponential growth to a maximum" type migration profile (Figure 1), reaching equilibrium during the first 48 hours of the assay, as previously reported by several works in the literature $[26,35]$. A proportional release was observed in TPC with the TPC present in the crude EOs: a higher release of TPC to the simulant media was observed for the EOs that showed the highest TPC, ginger and tea tree; and films incorporated
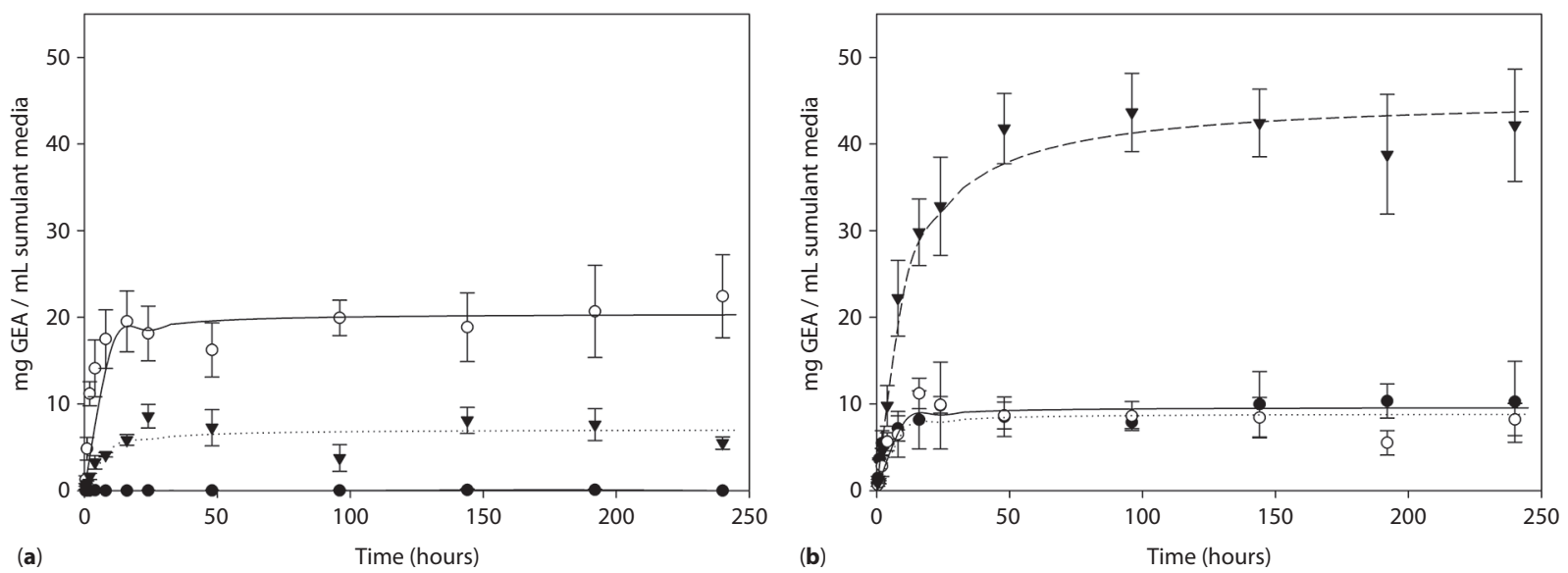

Figure 1 Total phenolic content of simulant media. Migration assay of chitosan films incorporated with different essential oils

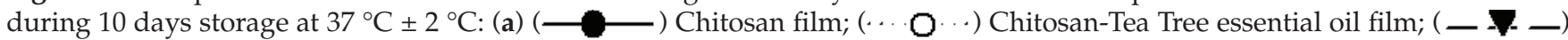
Chitosan-Rosemary essential oil film; (b) (__ Chitosan-Sage essential oil film; $(\cdots \mathbf{O} \cdots)$ Chitosan-Thyme essential oil film; ( — - C Chitosan-Ginger essential oil film. 
with rosemary, sage and thyme EO, which showed a lower TPC, also released a lower amount of phenolic compounds to the simulant. All the films, except the chitosan-ginger EO film, showed a similar release ratio between maximum phenolic content diffused and the total incorporated into the chitosan (0.34-0.52, Table 2). Chitosan-ginger EO film showed the highest release ratio, 0.92 (Table 2). It is known that the diffusion process, characteristic in migrations of additives and contaminants from packaging materials, is governed by the models based on Fick's second law (Eq. 2), which have been extensively used to provide estimations of the diffusion coefficients based on experimental data $[27,36]$.

Calculated diffusion coefficients are correlated with the release ratio of active compounds (Table 2), since ginger EO showed the highest coefficient $\left((5 \pm 1) \times 10^{-10}\right.$ $\left.\left(\mathrm{cm}^{2} . \mathrm{s}^{-1}\right)\right)$, followed by sage EO $\left((2.3 \pm 0.3) \times 10^{-10}\left(\mathrm{~cm}^{2} . \mathrm{s}^{-}\right.\right.$ $\left.\left.{ }^{1}\right)\right)$, thyme $\mathrm{EO}\left((1.5 \pm 0.4) \times 10^{-10}\left(\mathrm{~cm}^{2} \cdot \mathrm{s}^{-1}\right)\right)$, tea tree $\mathrm{EO}$ $\left((1.1 \pm 0.3) \times 10^{-10}\left(\mathrm{~cm}^{2} . \mathrm{s}^{-1}\right)\right)$ and rosemary EO $((0.6 \pm 0.1)$ $\left.\times 10^{-10}\left(\mathrm{~cm}^{2} \cdot \mathrm{s}^{-1}\right)\right)$. The findings are in good agreement with the results reported by Ramos et al. [37] that calculated the diffusion coefficient of carvacrol and thymol from polypropylene films in different simulant media. The authors reported values of $\mathrm{D}$ in the same magnitude: $2 \times 10^{-10}\left(\mathrm{~cm}^{2} \cdot \mathrm{s}^{-1}\right)$ and $1 \times 10^{-10}\left(\mathrm{~cm}^{2} \cdot \mathrm{s}^{-1}\right)$ for carvacrol and thymol at ethanol $95 \%$, respectively [37].

According to Jamshidian et al. [34], the fate of migration depends on several factors: (1) the characteristics of the polymer-its morphology, molecular weight, distribution, crystallinity, density, and orientation that may interfere with sizes, shapes and distribution of microcavities, therefore with diffusion path; (2) type of active compounds incorporated-its polarity, shape, chemical composition, solubility; (3) interaction polymer $\times$ active compounds incorporated-plasticizing and anticizing effects. Considering that the amount of glycerol and EOs incorporated were constant, the difference in the migration profile can only be explained by the type of active compounds incorporated, namely, its chemical composition, solubility and polarity. In the case of ginger EO, the higher release toward simulant media probably occurred due to a stronger solubility in ethanol $95 \%$. The lipophilic character of the ginger EOs active compounds was also suggested in the review made by Brewer [29] on natural antioxidants.

Antioxidant activity of the compounds diffused is shown in Figures 2 and 3. For the DPPH assay and ABTS assay, the antioxidant activity of the simulant media reached a maximum during the first 48 hours of the studies, following the pattern of the phenolic release. The only exception to this behavior was chitosan incorporated with rosemary EO. In this film, the antioxidant activity increased along the migration assay, reaching a maximum only at 192 hours (although the equilibrium is not well defined in the ABTS assay). In this case, the compounds with antioxidant activity presented a lower rate of diffusion to the simulant media than what was observed for the other EO films. According to Brewer [29], solvents with medium polarity, such as ethanol $50 \%$, are able to extract higher concentrations of compounds with antioxidant activity from rosemary EO than solvents with lower polarity, such as ethanol 95\%, which was used in the migration assay, explaining the slow rate of diffusion of the active compounds to the simulant. However, the ethanolic solution obtained in the chitosan-rosemary EO migration study showed the highest antioxidant activity, which correlated with the high antioxidant activity shown by rosemary EO. Phenolic diterpenes (carnosic acid, carnosol, rosmanol, rosmadial, 12-methoxycarnosic acid, epi- and isorosmanol) and phenolic acids (rosmarinic and caffeic) are the constituents of rosemary EO with the highest antioxidant activity [29]. A good relation between antioxidant activity of the EOs and antioxidant activity of the migration solution were also observed for the remaining films incorporated with EOs. The higher

Table 2 Migration results in chitosan-based films.

\begin{tabular}{|l|c|c|c|}
\hline Film & $\begin{array}{c}\text { Diffusion } \\
\text { coefficient }\left(\mathrm{cm}^{2} / \mathbf{s}\right)\end{array}$ & $\begin{array}{c}\text { Maximum total diffused/total } \\
\text { incorporated into films }\end{array}$ & $\begin{array}{c}\text { Maximum total antioxidant activity in the } \\
\text { simulant/total antioxidant activity in the film }\end{array}$ \\
\hline $\begin{array}{c}\text { Chitosan_Tea tree } \\
\text { essential oil film }\end{array}$ & $(1.1 \pm 0.3) \times 10^{-10}$ & 0.40 & 0.88 \\
\hline $\begin{array}{c}\text { Chitosan_Rosemary } \\
\text { essential oil film }\end{array}$ & $(0.6 \pm 0.1) \times 10^{-10}$ & 0.34 & 0.61 \\
\hline $\begin{array}{c}\text { Chitosan_Sage } \\
\text { essential oil film }\end{array}$ & $(2.3 \pm 0.3) \times 10^{-10}$ & 0.52 & 0.94 \\
\hline $\begin{array}{c}\text { Chitosan_Thyme } \\
\text { essential oil film }\end{array}$ & $(1.5 \pm 0.4) \times 10^{-10}$ & 0.41 & 1.0 \\
\hline $\begin{array}{c}\text { Chitosan_Ginger } \\
\text { essential oil film }\end{array}$ & $(5 \pm 1) \times 10^{-10}$ & 0.92 & \\
\hline
\end{tabular}



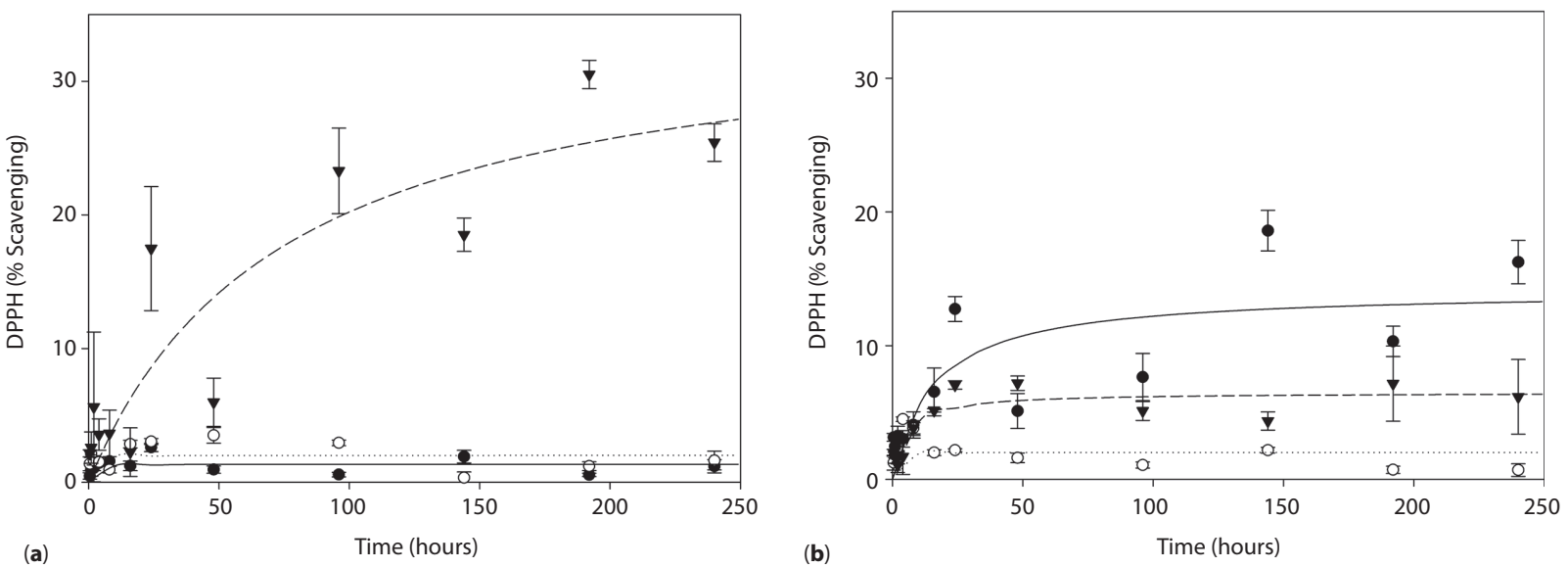

Figure 2 Antioxidant activity of simulant media expressed in DPPH by scavenging percentage. Migration assay of chitosan

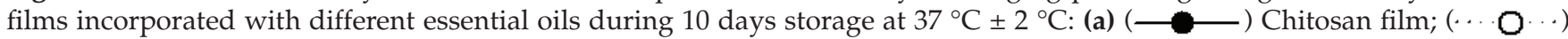
Chitosan-Tea Tree essential oil film; ( _ _ _ Chitosan-Rosemary essential oil film; (b) (_- - C Chitosan-Sage essential oil film; $(\cdots \mathrm{O} \cdots)$ Chitosan-Thyme essential oil film; ( $-\boldsymbol{\nabla}$ - Chitosan-Ginger essential oil film.
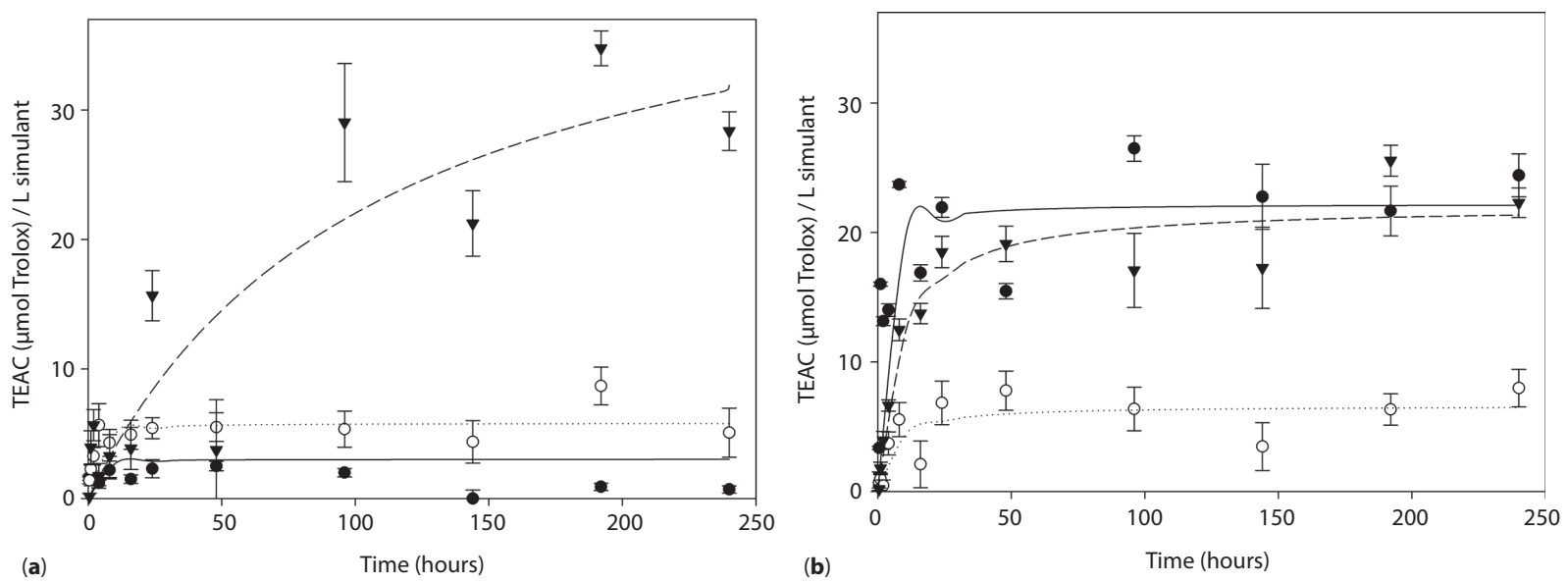

Figure 3 Antioxidant activity of simulant media expressed in Trolox equivalent (TEAC). Migration assay of chitosan films incorporated with different essential oils during 10 days storage at $37^{\circ} \mathrm{C} \pm 2{ }^{\circ} \mathrm{C}$ : (a) (—- Chitosan film; $(\cdots \mathrm{O} \cdots)$ ChitosanTea Tree essential oil film; (_- _ C Chitosan-Rosemary essential oil film; (b) (_$(\cdots \mathrm{O} \cdots)$ Chitosan-Thyme essential oil film; ( $-\boldsymbol{\nabla} \ldots)$ Chitosan-Ginger essential oil film.

the antioxidant activity of the Eos, the higher the antioxidant activity observed in the migration solution (as was observed for rosemary EO in the DPPH and ABTS assays and also for ginger and sage EOs in the ABTS assay); and the lower the antioxidant activity of the Eos, the lower the antioxidant activity observed in the migration solution (as was observed for thyme and tea tree EOs) (Figures 2 and 3). The exception was ginger EO films in the DPPH assay. In this case, ginger EO presented a high antioxidant activity (similar to the one presented by rosemary EO), but in the simulant media it scavenges only $5 \%$ of the DPPH radicals, much lower than the $25 \%$ observed for rosemary EO (Figure 2). Ginger antioxidant activity may be attributed to the presence of phenolic acids (caffeic and gallic) and volatiles (e.g., $\alpha$ - and $\beta$-pinene, gingerol, $\mathrm{p}$-cymene) [29]. Sage antioxidant activity is linked with some of the same compounds found in rosemary (rosmanol, rosmadial, carnosol, carnosic acid and rosmarinic acid) along with flavonoids [29], and also with $\alpha$ - and $\beta$-pinene and 1,8-cineole [38]. Concerning thyme, the antioxidant compounds associated with it are thymol (the most effective) and other volatiles such as carvacrol, $\alpha$ - and $\beta$-pinene, 1,8-cineole, p-cymene and $\alpha$-thujene, the phenolic acid, rosmarinic acid, and flavonoids $[29,38]$. The phenolic compounds found in tea tree are terpinen-4-ol, $\alpha$ - and $\gamma$-terpine and 1,8-cineole, but only the last has antioxidant activity [39].

All the films, except the chitosan-rosemary EO film, in terms of antioxidant activity measured through the 
ABTS assay, showed a similar result for the maximum diffused or total incorporated into the chitosan (0.841.00, Table 2). Chitosan-rosemary EO film showed the lowest ratio, 0.61 (Table 2). Yet, this lower diffusion of compounds with antioxidant activity can be due to the slower release of the phenols from the chitosan matrix due to the low polarity of the ethanol $95 \%$, as explained before. It also should be mentioned that the phenolic compounds released to the simulant media remained stable and did not degrade during the migration test (10 days) and no significant loss of volatile antioxidants was observed during the drying of the films $(<5 \%)$. In fact, the method used to produce the films was chosen to avoid/prevent those losses.

\subsection{Attenuated Total Reflectance Fourier Transform Infrared Spectroscopy (ATR-FTIR)}

The ATR-FTIR spectra of chitosan films incorporated with natural antioxidants from different sources is shown in Figure 4.

In general, all spectra were similar since the amount of active compounds incorporated (1\%) was small and

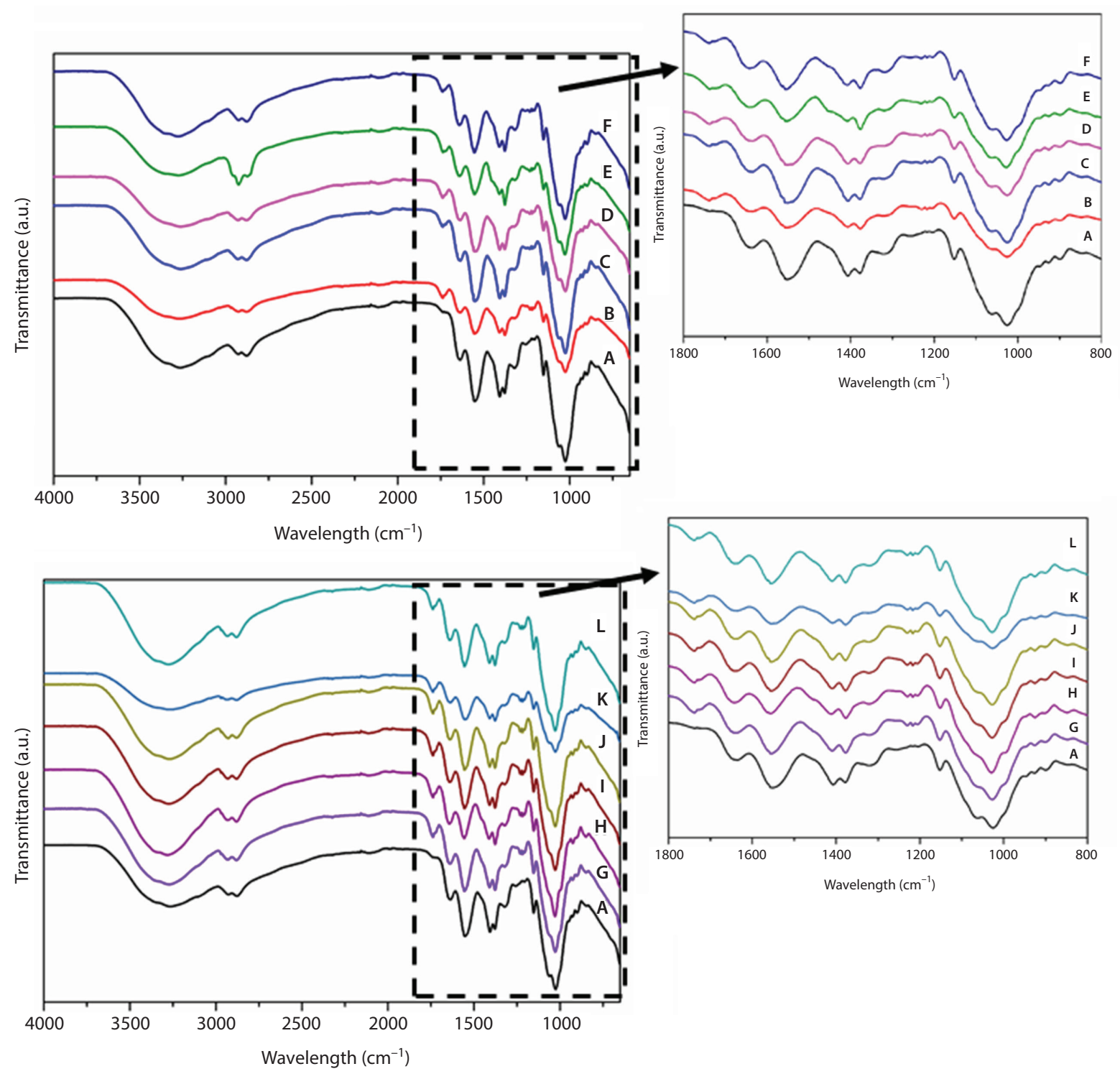

Figure 4 ATR-FTIR spectra of chitosan (Ch)-based films. (A) Neat chitosan film; (B) Chitosan-Tea Tree essential oil film; (C) Chitosan-Rosemary essential oil film; (D) Chitosan-Sage essential oil film; (E) Chitosan-Ginger essential oil film; (F) ChitosanThyme essential oil film; (G) Chitosan-Sage hydro-alcoholic extract film; (H) Chitosan-Kenaf hydro-alcoholic extract film; (I) Chitosan-Ginger hydro-alcoholic extract film; (J) Chitosan-Black Tea hydro-alcoholic extract film; (K) Chitosan-Green Tea hydro-alcoholic extract film; (L) Chitosan-Rosemary hydro-alcoholic extract film. 
ATR mode was used. Infrared spectra of pristine chitosan films exhibited characteristic absorption bands at $3332 \mathrm{~cm}^{-1}$ (-OH asymmetric stretching); $3246 \mathrm{~cm}^{-1}$ (-NH asymmetric stretching); $2867 \mathrm{~cm}^{-1}$ (C-H bonding of $-\mathrm{NHCOCH}_{3}$ metal group); $1635 \mathrm{~cm}^{-1}$ (amide I); $1550 \mathrm{~cm}^{-1}$ (vibration - $\mathrm{NH}_{2}$ - amide II); $1337 \mathrm{~cm}^{-1}$ (skeleton vibration involving $\mathrm{C}-\mathrm{N}$ stretching of amide III) and 1376-1404 $\mathrm{cm}^{-1}$ (- $\mathrm{CH}_{2}$ bending) [40-42]. Also, the peaks at $922 \mathrm{~cm}^{-1}, 1022 \mathrm{~cm}^{-1}$ and $1047 \mathrm{~cm}^{-1}$ (skeletal vibrations involving the $\mathrm{C}-\mathrm{O}$ stretching) and $1150 \mathrm{~cm}^{-1}$ (asymmetric stretching of the $\mathrm{C}-\mathrm{O}-\mathrm{C}$ bridge) are characteristic of the saccharide structure of chitosan [41].

The incorporation of natural antioxidants provoked changes in the absorption spectra between bands $1200 \mathrm{~cm}^{-1}$ and $1400 \mathrm{~cm}^{-1}$ due to the hydroxyl groups of phenolic compounds and the $\mathrm{C}-\mathrm{O}$ and $\mathrm{C}=\mathrm{O}$ vibrations [25, 41, 42]. Absorption at $1550 \mathrm{~cm}^{-1}$ (amide II) decreased, especially with the incorporation of ginger and tea tree EOs and kenaf and green tea HAEs, suggesting that chitosan amide group bonded with the polyphenols present in the EOs and HAEs tested [42].

The new peak at $1715-1730 \mathrm{~cm}^{-1}$ that was seen in all the spectra with OEs and HAESs, was attributed to an ester bond between carbonyl groups of phenolic acids and chitosan, as suggested by Silva-Weiss et al. [42] and Siripatrawan and Harte [25]. The absorption reduction observed between 3500 and $3000 \mathrm{~cm}^{-1}$ for some of the films incorporated with EOs or HAEs (namely green tea HAE and ginger, tea tree and sage EOs) confirms the linkage between phenolic compounds and chitosan, as these bands correspond to stretching of free hydroxyl and to asymmetric and symmetric stretching of the N-H bonds in amino group. This interaction between polyphenolic hydroxyl groups and chitosan amide groups results in improvements in mechanical and barrier properties [19, 25].

\section{CONCLUSIONS}

Chitosan films were successfully casted with incorporation of different natural compounds, either hydroalcoholic extracts or essential oils, resulting in homogeneous materials for further use in the food industry. The successful incorporation and interaction between the polymeric matrices and the phenolic compounds was observed through migration assays, especially for films incorporated with HAEs, where no diffusion was observed for all the extracts tested. The good interaction between phenolic compounds from HAEs and chitosan can protect the food packaged by other mechanisms, such as acting as an improved barrier against light, water and oxygen from the outer environment. On the other hand, biopolymers added with EO showed exponential diffusion growth, and the active compounds present at the simulant kept its antioxidant activity. EOs showed a faster release toward fatty food simulant, highlighting their use for this type of product. Regarding the antioxidant potential verified in the simulant media, films incorporated with ginger, sage and rosemary EOs presented the best results.

In order to rank the different HAEs and EOs for further incorporation into chitosan for food packaging, further studies are demanded, namely the identification of the antimicrobial activity of the novel materials and their behavior when in direct contact with food matrices.

\section{ACKNOWLEDGMENTS}

This work was supported by CNPq/Brazil (grant number 200790/2014-5); CAPES (APQ-1 2009/02 E-26/110.414/2010, APQ-1 2011-2 E-26/110.269.2012, E-26/111.435/2012 - CsF/ Brazil - BEX 11943-13-0); CENIMAT/I3N and MEtRiCS through FCT/MCTES (project numbers UID/CTM/50025/2013 and UID/ SEM/04077/2013CN).

\section{REFERENCES}

1. A. Wojdyło, J. Oszmiański, and R. Czemerys, Antioxidant activity and phenolic compounds in 32 selected herbs. Food Chem. 105, 940-949 (2007). DOI: 10.1016/j. foodchem.2007.04.038

2. A. Vallverdú-Queralt, J. Regueiro, M. MartínezHuélamo, J.F. Rinaldi Alvarenga, L.N. Leal, and R.M. Lamuela-Raventos, A comprehensive study on the phenolic profile of widely used culinary herbs and spices: Rosemary, thyme, oregano, cinnamon, cumin and bay. Food Chem. 154, 299-307 (2014). DOI: 10.1016/j. foodchem.2013.12.106

3. B. Shan, Y.Z. Cai, M. Sun, and H. Corke, Antioxidant capacity of 26 spice extracts and characterization of their phenolic constituents. J. Agric. Food Chem. 53, 7749-7759 (2005). DOI: 10.1021/jf051513y

4. A. Pascoal, R. Quirantes-Piné, A.L. Fernando, E. Alexopoulou, and A. Segura-Carretero, Phenolic composition and antioxidant activity of kenaf leaves. Ind. Crops Prod. 78, 116-123 (2015). DOI: 10.1016/j. indcrop.2015.10.028

5. R. Ribeiro-Santos, D. Carvalho-Costa, C. Cavaleiro, H.S. Costa, T.G. Albuquerque, M.C. Castilho, F. Ramos, N.R. Melo, and A. Sanches-Silva, A novel insight on an ancient aromatic plant: The rosemary (Rosmarinus officinalis L.). Trends Food Sci. Technol. 45, 355-368 (2015). DOI: 10.1016/j.tifs.2015.07.015

6. A. Sanches-Silva, D. Costa, T.G. Albuquerque, G.G. Buonocore, F. Ramos, M.C. Castilho, A.V. Machado, and H.S. Costa, Trends in the use of natural antioxidants in active food packaging: A review. Food Addit. Contam. Part A 31, 374-395 (2014). DOI: 10.1080/19440049.2013.879215 
7. Z.A. Maryam Adilah and Z.A. Nur Hanani, Active packaging of fish gelatin films with Morinda citrifolia oil. Food Biosci. 16, 66-71 (2016). DOI: 10.1016/j.fbio.2016.10.002

8. J. Gómez-Estaca, C. López-de-Dicastillo, P. HernándezMuñoz, R. Catalá, and R. Gavara, Advances in antioxidant active food packaging. Trends Food Sci. Technol. 35, 42-51 (2014). DOI: 10.1016/j.tifs.2013.10.008

9. C.C. Pola, E.A.A. Medeiros, O.L. Pereira, V.G.L. Souza, C.G. Otoni, G.P. Camilloto, and N.F.F. Soares, Cellulose acetate active films incorporated with oregano (Origanum vulgare) essential oil and organophilic montmorillonite clay control the growth of phytopathogenic fungi. Food Packag. Shelf Life 9, 69-78 (2016). DOI: 10.1016/j. fpsl.2016.07.001

10. C.G. Otoni, S.F.O. Pontes, E.A.A. Medeiros, and N.D.F.F. Soares, Edible films from methylcellulose and nanoemulsions of clove bud (Syzygium aromaticum) and oregano (Origanum vulgare) essential oils as shelf life extenders for sliced bread. J. Agric. Food Chem. 62, 5214-5219 (2014). DOI: 10.1021/jf501055f

11. C. Nerín, L. Tovar, and J. Salafranca, Behaviour of a new antioxidant active film versus oxidizable model compounds. J. Food Eng. 84, 313-320 (2008). DOI: 10.1016/j. jfoodeng.2007.05.027

12. T. Bolumar, M.L. Andersen, and V. Orlien, Antioxidant active packaging for chicken meat processed by high pressure treatment. Food Chem. 129, 1406-1412 (2011). DOI: 10.1016/j.foodchem.2011.05.082

13. V.G.L. Souza and A.L. Fernando, Nanoparticles in food packaging: Biodegradability and potential migration to food-A review. Food Packag. Shelf Life 8, 63-70 (2016). DOI: 10.1016/j.fpsl.2016.04.001

14. M. Darder, M. Colilla, and E. Ruiz-Hitzky, Biopolymerclay nanocomposites based on chitosan intercalated in montmorillonite. Chem. Mater. 15, 3774-3780 (2003). DOI: $10.1021 / \mathrm{cm} 0343047$

15. U.Siripatrawan and S. Noipha, Active film from chitosan incorporating green tea extract for shelf life extension of pork sausages. Food Hydrocoll. 27, 102-108 (2012). DOI: 10.1016/j.foodhyd.2011.08.011

16. X.Z. Tang, P. Kumar, S. Alavi, and K.P. Sandeep, Recent advances in biopolymers and biopolymerbased nanocomposites for food packaging materials. Crit. Rev. Food Sci. Nutr. 52, 426-442 (2012). DOI: 10.1080/10408398.2010.500508

17. F. Tian, E.A. Decker, and J.M. Goddard, Controlling lipid oxidation of food by active packaging technologies. Food Funct. 4, 669-680 (2013). DOI: 10.1039/c3fo30360h

18. C. Pastor, L. Sánchez-González, A. Chiralt, M. Cháfer, and C. González-Martínez, Physical and antioxidant properties of chitosan and methylcellulose based films containing resveratrol. Food Hydrocoll. 30, 272-280 (2013). DOI: 10.1016/j.foodhyd.2012.05.026

19. V.G.L. Souza, A.L. Fernando, J.R.A. Pires, P.F. Rodrigues, A.A.S. Lopes, and F.M.B. Fernandes, Physical properties of chitosan films incorporated with natural antioxidants. Ind. Crops Prod. 107, 565-572 (2017). DOI: 10.1016/j. indcrop.2017.04.056

20. N. Turkmen, F. Sari, and Y.S. Velioglu, Effects of extraction solvents on concentration and antioxidant activity of black and black mate tea polyphenols determined by ferrous tartrate and Folin-Ciocalteu methods. Food Chem. 99, 835-841 (2006). DOI: 10.1016/j.foodchem.2005.08.034

21. V.L. Singleton, R. Orthofer, and R.M. Lamuela-Raventós, Analysis of total phenols and other oxidation substrates and antioxidants by means of Folin-Ciocalteu. Methods Enzymol. 299, 152-178 (1999).

22. W. Brand-Williams, M.E. Cuvelier, and C. Berset, Use of a free radical method to evaluate antioxidant activity. LWT-Food Sci. Technol. 28, 25-30 (1995).

23. R. Re, N. Pellegrini, A. Proteggente, A. Pannala, M. Yang, and C. Rice-Evans, Antioxidant activity applying an improved ABTS radical. Free Radical. Biol. Med. 26, 1231-1237 (1999).

24. M. Abdollahi, M. Rezaei, and G. Farzi, A novel active bionanocomposite film incorporating rosemary essential oil and nanoclay into chitosan. J. Food Eng. 111, 343-350 (2012). DOI: 10.1016/j.jfoodeng.2012.02.012

25. U. Siripatrawan and B.R. Harte, Physical properties and antioxidant activity of an active film from chitosan incorporated with green tea extract. Food Hydrocoll. 24, 770-775 (2010). DOI: 10.1016/j.foodhyd.2010.04.003

26. C. López-de-Dicastillo, J. Gómez-Estaca, R. Catalá, R. Gavara, and P. Hernández-Muñoz, Active antioxidant packaging films: Development and effect on lipid stability of brined sardines. Food Chem. 131, 376-1384 (2012). DOI: $10.1016 /$ j.foodchem.2011.10.002

27. D. Chung, S.E. Papadakis, and K.L. Yam, Simple models for assessing migration from food-packaging films. Food Addit. Contam. 19, 611-617 (2002). DOI: 10.1080/0265203021012638

28. G. Rusak, D. Komes, S. Likić, D. Horžić, and M. Kovač, Phenolic content and antioxidative capacity of green and white tea extracts depending on extraction conditions and the solvent used. Food Chem. 110, 852-858 (2008). DOI: $10.1016 /$ j.foodchem.2008.02.072

29. M.S. Brewer, Natural antioxidants: Sources, compounds, mechanisms of action, and potential applications. Compr. Rev. Food Sci. Food Saf. 10, 221-247 (2011). DOI: 10.1111/j.1541-4337.2011.00156.x

30. H. Liu, N. Qiu, H. Ding, and R. Yao, Polyphenols contents and antioxidant capacity of 68 Chinese herbals suitable for medical or food uses. Food Res. Int. 41, 363-370 (2008). DOI: 10.1016/j.foodres.2007.12.012

31. M.P. Almajano, R. Carbó, J.A.L. Jiménez, and M.H. Gordon, Antioxidant and antimicrobial activities of tea infusions. Food Chem. 108, 55-63 (2008). DOI: 10.1016/j.foodchem.2007.10.040

32. E.W.C. Chan, Y.Y. Lim, K.L. Chong, J.B.L. Tan, and S.K. Wong, Antioxidant properties of tropical and temperate herbal teas. J. Food Compos. Anal. 23, 185-189 (2010). DOI: 10.1016/j.jfca.2009.10.002

33. M.E.I. Badawy and S.A.M. Abdelgaleil, Composition and antimicrobial activity of essential oils isolated from Egyptian plants against plant pathogenic bacteria and fungi. Ind. Crops Prod. 52, 776-782 (2014). DOI: 10.1016/j. indcrop.2013.12.003

34. M. Jamshidian, E.A. Tehrany, and S. Desobry, Antioxidants release from solvent-cast PLA film: Investigation of PLA antioxidant-active packaging. Food 
Bioprocess Technol. 6, 1450-1463 (2012). DOI: 10.1007/ s11947-012-0830-9

35. M. Ramos, A. Jiménez, M. Peltzer, and M.C. Garrigós, Development of novel nano-biocomposite antioxidant films based on poly (lactic acid) and thymol for active packaging. Food Chem. 162, 149-155 (2014). DOI: 10.1016/j.foodchem.2014.04.026

36. E. Fortunati, M. Peltzer, I. Armentano, A. Jiménez, and J.M. Kenny, Combined effects of cellulose nanocrystals and silver nanoparticles on the barrier and migration properties of PLA nano-biocomposites. J. Food Eng. 118, 117-124 (2013). DOI: 10.1016/j. jfoodeng.2013.03.025

37. M. Ramos, A. Beltrán, M. Peltzer, A.J.M. Valente, abd M.D.C. Garrigós, Release and antioxidant activity of carvacrol and thymol from polypropylene active packaging films. LWT - Food Sci. Technol. 58, 470-477 (2014). DOI: 10.1016/j.lwt.2014.04.019

38. S. Burt, Essential oils: Their antibacterial properties and potential applications in foods - A review. Int.
J. Food Microbiol. 94, 223-253 (2004). DOI: 10.1016/j. ijfoodmicro.2004.03.022

39. C.F. Carson, K.A. Hammer, and T.V. Riley, Melaleuca alternifolia (tea tree) oil: A review of antimicrobial and other medicinal properties. Clin. Microbiol. Rev. 19, 50-62 (2006). DOI: 10.1128/CMR.19.1.50-62.2006

40. K.W. Kim and R. Thomas, Antioxidative activity of chitosans with varying molecular weights. Food Chem. 101,308-313 (2007). DOI: 10.1016/j.foodchem.2006.01.038

41. A. Ávila, K. Bierbrauer, G. Pucci, M. López-González, and M. Strumia, Study of optimization of the synthesis and properties of biocomposite films based on grafted chitosan. J. Food Eng. 109, 752-761 (2012). DOI: 10.1016/j. jfoodeng.2011.11.009

42. A. Silva-Weiss, V. Bifani, M. Ihl, P.J.A. Sobral, and M.C. Gómez-Guillén, Structural properties of films and rheology of film-forming solutions based on chitosan and chitosan-starch blend enriched with murta leaf extract. Food Hydrocoll. 31, 458-466 (2013). DOI: 10.1016/j.foodhyd.2012.11.028 